\title{
A Is for Amyloid
}

\author{
D.J. Selkoe
}

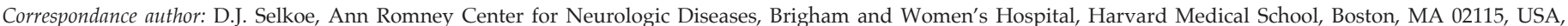
dselkoe@bwh.harvard.edu

J Prev Alz Dis 2020;3(7):140-141

Published online April 28, 2020, http:/ /dx.doi.org/10.14283/jpad.2020.27

I n the age of COVID-19, we are reminded that despite the enormous strides modern medicine has made against acute infectious pathogens, we can still be overwhelmed. And in the field of chronic non-infectious diseases of the brain, we, too, have been traveling a long and unpredictable road. For years, there has been a sense of pessimism about the halting march toward disease-modifying treatments for Alzheimer's disease. But recent events may have begun to part the clouds. In this issue of JPAD, Aisen et al. (1), representing the EU/US CTAD 2019 Task Force, provide a thoughtful perspective on progress in certain anti-amyloid trials and the resultant lessons for our next steps toward success.

The whiff of optimism emerges principally from a reanalysis of EMERGE and ENGAGE, Biogen's Phase 3 trials of the human $A \beta$ antibody, aducanumab. After what is now recognized as a flawed futility analysis of just half of the trial participants, Biogen reported at CTAD last December 5 that analyzing all 3,285 patients with very mild or mild $A D$ revealed that EMERGE met its primary and secondary endpoints, including significantly less decline on two cognitive tests and on a measure of activities of daily living, whereas ENGAGE missed its endpoints but a post-hoc analysis of those receiving the highest exposure $(10 \mathrm{mg} / \mathrm{kg} / \mathrm{mo}$ for at least 10 months) also showed less decline on these measures. Concerns were immediately raised that the effect sizes were rather modest. But the argument that they are no greater than those of symptomatic agents like donepezil misses the point: the latter have no disease-modifying activity and often lose efficacy within months, whereas aducanumab cannot affect symptoms acutely but actually slows the pathogenic process (vide infra), suggesting it may gain efficacy over time.

Although clinical meaningfulness is the key outcome all of us (including regulators) seek, the striking biomarker benefits in the aducanumab trials may be the real story. That is because both ENGAGE and EMERGE produced impressive evidence of not only robustly lowering amyloid plaque burden but also decreasing CSF phosphotau levels and brain tau-PET signal, two indicators that reduced tangle burden had occurred. This combination provides biological proof of disease modification and is in line with what the amyloid hypothesis had long predicted: $A \beta$ species, especially soluble oligomers, bind to neuritic membranes in a way that induces the hyperphosphorylation and insolublization of tau. And the story got even better when April arrived and the (virtual) AAT-AD/PD meeting unfolded. There, reports of the clinically negative DIANTU trials of solanezumab and gantenerumab in familial AD subjects revealed a robust lowering of amyloid PET by the latter antibody, accompanied by an important decrease in CSF ptau and brain tau-PET. Once again, we witnessed biological evidence of modifying the otherwise inexorable progression of Alzheimer's in the brain. Consistent with these two reports is earlier positive data in a Phase $2 \mathrm{~b}$ trial of BAN2401, announced in 2018. This antibody to synthetic $A \beta$ protofibrils (large filamentous oligomers) infused at a high dose of $10 \mathrm{mg} /$ $\mathrm{kg}$ biweekly produced both encouraging clinical signals and biomarkers (including amyloid-PET and CSF ptau) headed in the right direction.

Taken together, the extant biomarker results of aducanumab, BAN2401 and gantenerumab signify that at least some $A \beta$ antibodies can reduce the two key neuropathological lesions which define $A D$, plaques and then tangles. This objective support for the amyloid hypothesis should be viewed with excitement, because it means that the AD field has achieved biological disease modification. Now, we need to do this even more effectively to bring along real clinical benefit to our patients. But what does "more effectively" mean? First, it means choosing an antibody that can clear amyloid plaques and thereby decrease oligomer burden, since $\mathrm{AD}$ plaques have been proven to contain synaptotoxic oligomers (e.g., 2, 3). Plaque-clearing antibodies are simultaneously able to bind and neutralize soluble oligomers that injure neurons and activate microglia and astrocytes. Second, we need to dose plaque/oligomerclearing antibodies as high as possible, as long as adverse events are manageable. As Aisen et al. (1) emphasize: dose matters, and the only "serious" adverse event in such trials is ARIA-E, which is not a show stopper; it is mostly asymptomatic and almost always self-limited. Third, we need to treat this insidious, chronic disease for as long as we can, because cumulative plaque/oligomer lowering and tangle prevention is likely to lead gradually to greater clinical benefit. Fourth, we should initiate such antibodies as early as we can, at the onset of subtle 
cognitive symptoms or years before that.

These four principles emerging from our recent successes provide a roadmap for testing (and after approval, prescribing) $\mathrm{AD}$-slowing $\mathrm{A} \beta$ antibodies. The 3 antibodies highlighted above are worthy of even more well-designed trials, following the guidelines Aisen et al. (and others) have articulated. But we should also search for even better oligomer neutralizing/clearing antibodies, and there are ways to quantify their efficacy preclinically by applying them to iPSC-derived human neurons exposed to brain-derived soluble $A \beta$ oligomers (4), followed by trials in knock-in mouse models that most closely reflect the human disease state. At this juncture, high doses and long durations may matter more than finding the "perfect" antibody. The latter references a question I am often asked: which are the most toxic oligomers? Further biochemical studies of human $A \beta$ isolates applied to human neurons may reveal answers, but I suspect there is no "super-oligomer". Rather, different sizes and conformations of oligomers built from dimers (the "first" oligomer) that then bind to plasma membranes and grow dynamically in vivo may be particularly adverse biophysically. We don't need to await better molecular definition of the most noxious oligomers; we can use certain oligomer-targeting antibodies to activate amyloid-clearing mechanisms, e.g. microglia, and thus enable "bystander clearance" of many A $\beta$ forms.

Our field has divided opinions on whether the aducanumab data are worthy of some type of "accelerated" or "conditional" approval as the first disease-modifying therapeutic for AD. There are arguments on both sides. Requiring a third pivotal trial to justify approval would avoid releasing an agent with an apparently modest clinical effect. But in my view, EMERGE, when coupled with the high dose/long duration data of ENGAGE, signifies that an agent which clearly moves AD biology and acts significantly better than placebo clinically warrants a regulated approval for very mild $\mathrm{AD}$ patients resembling those who were helped in these trials. A positive decision will strongly encourage more antibody trials, enable the necessary development of $A \beta$ vaccines, and provide the first step toward combination therapy with a tau-lowering biologic. Many thousands of patients have made heroic efforts to bring us this far, and we need to begin thoughtfully treating Alzheimer's disease in the real world.

\section{References}

1. P.S. Aisen, J. Cummings, R. Doody, L. Kramer, S. Salloway, D.J. Selkoe, J. Sims, R.A. Sperling, B. Vellas and the EU/US CTAD 2019 Task Force*. The Future of Anti-Amyloid Trials. J Prev Alz Dis 2020;3(7):146-151.

2. Shankar GM1, Li S, Mehta TH, Garcia-Munoz A, Shepardson NE, Smith I, Brett FM, Farrell MA, Rowan MJ, Lemere CA, Regan CM, Walsh DM, Sabatini BL, Selkoe DJ. Amyloid-beta protein dimers isolated directly from Alzheimer's brains impair synaptic plasticity and memory. Nat Med. 2008 Aug;14(8):837-42. doi: 10.1038/nm1782. Epub 2008 Jun 22.

3. Esparza TJ1, Zhao H, Cirrito JR, Cairns NJ, Bateman RJ, Holtzman DM, Brody DL. Amyloid- $\beta$ oligomerization in Alzheimer dementia versus high-pathology controls. Ann Neurol. 2013 Jan;73(1):104-19. doi: 10.1002/ ana.23748. Epub 2012 Dec 7.

4. Jin M, O'Nuallain B, Hong W, Boyd J, Lagomarsino VN, O'Malley TT, Liu W, Vanderburg CR, Frosch MP, Young-Pearse T, Selkoe DJ, Walsh DM. An in vitro paradigm to assess potential anti-aß antibodies for Alzheimer's disease. Nat Commun 2018; Jul 11; 9(1)2676. PMCID: PMC6041266 